\title{
Highly integrated photonic crystal bandedge lasers monolithically grown on Si substrates
}

\author{
Yaoran Huang (黄要然 ${ }^{1, \dagger}$ ， Taojie Zhou (周陶杰) ${ }^{1,2, \dagger}$, Mingchu Tang (唐明初) ${ }^{2}$, Guohong Xiang (项国洪) ${ }^{1}$, \\ Haochuan Li (李浩川) $)^{1}$, Mickael Martin ${ }^{3}$, Thierry Baron ${ }^{3}$, Siming Chen (陈思铭) ${ }^{2}$, \\ Huiyun Liu (刘会望武) ${ }^{2}$, and Zhaoyu Zhang (张昭宇) $)^{1^{*}}$ \\ ${ }^{1}$ School of Science and Engineering and Shenzhen Key Laboratory of Semiconductor Lasers, The Chinese University of Hong Kong, Shenzhen (CUHKSZ), \\ Shenzhen 518172 , China \\ ${ }^{2}$ Department of Electronic and Electrical Engineering, University College London, London WCIE 7JE, UK \\ 3 Université Grenoble Alpes, CNRS, CEA-LETI, MINATEC, LTM, F-38054 Grenoble, France
}

*Corresponding author: zhangzy@cuhk.edu.cn

Received November 16, 2021 | Accepted January 14, 2022 | Posted Online February 16, 2022

\begin{abstract}
Monolithic integration of III-V lasers with small footprint, good coherence, and low power consumption based on a CMOScompatible Si substrate have been known as an efficient route towards high-density optical interconnects in the photonic integrated circuits. However, the material dissimilarities between Si and III-V materials limit the performance of monolithic microlasers. Here, under the pumping condition of a continuous-wave $632.8 \mathrm{~nm}$ He-Ne gas laser at room temperature, we achieved an InAs/GaAs quantum dot photonic crystal bandedge laser, which is directly grown on an on-axis Si (001) substrate, which provides a feasible route towards a low-cost and large-scale integration method for light sources on the Si platform.
\end{abstract}

Keywords: lasers; bandedge; photonic crystal; monolithic integration; quantum dots; silicon substrate.

DOI: 10.3788/COL202220.041401

\section{Introduction}

Advanced microscale silicon photonics technology has emerged as a promising candidate for the next-generation chip-scale data communication network due to its unique advantages of low cost, high integration density, high speed, and energy efficiency $^{[1,2]}$. However, a highly efficient microscale Si-based light source is still considered as the obstacle for realizing a practical Si-based photonic integrated circuit (PIC), due to the indirect bandgap nature of bulk $\mathrm{Si}$ and Ge materials.Various approaches towards integration of microcavity lasers on silicon substrates have been demonstrated extensively, including co-packing, hybrid integration, wafer bonding, etc. But, these methods more or less include cumbersome fabrication processes ${ }^{[1,2]}$. The monolithic integration method, however, is another promising route towards low cost and scalable Si-based PICs. Monolithic integration takes advantage of the CMOS-compatible fabrication process of contemporary integrated circuits. It is unnecessary to consider heterogenous methods to make precise alignment between the light souce and other optical components. Nevertheless, the fundamental challenge of epitaxial III-V on $\mathrm{Si}$ is the degraded material quality caused by large material dissimilarities ${ }^{[3]}$. The planar defect and anti-phase boundary, due to the polar on non-polar growth, can be avoided by employing a pretreated double-atomic-step Si substrate ${ }^{[4]}$. Furthermore, a strained-layer superlattice (SLS) has been used as defect filter layers (DFLs) to significantly reduce the threading defect caused by the lattice mismatched heteroepitaxial growth of III-V materials on $\mathrm{Si}^{[5]}$. Those works pave the way for highperformance ridge-waveguide lasers with quantum dot (QD) gain medium directly grown on the group-IV substrates through various intermediate buffer layers and offcut substrates, including $\mathrm{Ge}^{[6]}$, Ge-on-Si ${ }^{[7]}, \mathrm{GaP} / \mathrm{Si}(001)^{[8]}$, patterned on-axis Si $(001)^{[9]}$, and Si substrate with a $4^{\circ}$ offcut angle ${ }^{[3]}$. However, the relatively large footprint of the ridge-waveguide laser limits the realization of microscale Si-based PICs. Recently, we have demonstrated InAs/GaAs QD microdisk lasers and photonic crystal membrane lasers monolithically grown on CMOScompatible on-axis Si (001) substrates ${ }^{[10-12]}$.

Meanwhile, defect-free photonic crystal bandedge laser arrays enable a large-area coherent oscillation, so a large number of attractive applications have been derived ${ }^{[13,14]}$, including laser-based processing and light detection. The group velocity of high symmetry points in the energy band diagram can be very low. When a mode with group velocity is close to zero in the material gain region, the bandedge effect occurs. There is a large increase in optical path length for the bandedge point, which significantly improves the effective gain. Various 
high-performance photonic crystal bandedge surface emitting lasers have been demonstrated with high output power and single mode emission ${ }^{[15,16]}$.

In this paper, we demonstrate InAs/GaAs QD square lattice photonic crystal bandedge lasers monolithically grown on an on-axis $\mathrm{Si}$ (001) substrate with ultra-low lasing threshold under $632.8 \mathrm{~nm} \mathrm{He}-\mathrm{Ne}$ gas laser source at room temperature. This square lattice photonic crystal bandedge laser on $\mathrm{Si}$ can be a promising candidate for the coherent light source for Si photonics due to its low threshold characteristic and potential capability for high-power output.

\section{Device Fabrication}

The doping-free CMOS-compatible on-axis $\mathrm{Si}$ (001) substrates are offered for the monolithic growth of InAs/GaAs QD photonic crystal bandedge lasers ${ }^{[1,12]}$. The as-grown $400 \mathrm{~nm}$ GaAs layer is on the on-axis $\mathrm{Si}(001)$ substrate with a $0.15^{\circ}$ misorientation with two steps by metalorganic chemical vapor deposition, and then the substrate is transferred to the molecular beam epitaxy (MBE) chamber for subsequent material growth. Four sets of optimized DFLs are grown after a $200 \mathrm{~nm}$ of GaAs buffer layer, which is grown at $580^{\circ} \mathrm{C}$ to make the epitaxial surface smooth, while each set of DFLs contains a low-temperature growth of $\operatorname{In}_{0.18} \mathrm{Ga}_{0.82} \mathrm{As} / \mathrm{GaAs}$ SLSs at $450^{\circ} \mathrm{C}$, followed by an in-situ thermal annealing at $600^{\circ} \mathrm{C}$ for $5 \mathrm{~min}$, and then a

(a)

$$
\begin{aligned}
& 40 \mathrm{~nm} \mathrm{Al}_{0.4} \mathrm{GaAs} \text { cladding layer } \\
& 50 \mathrm{~nm} \text { GaAs } \\
& \text { QDs in } 8 \mathrm{~nm} \ln _{0.15} \mathrm{Ga}_{0.85} \text { As } \\
& 50 \mathrm{~nm} \text { GaAs }
\end{aligned}
$$

(d)

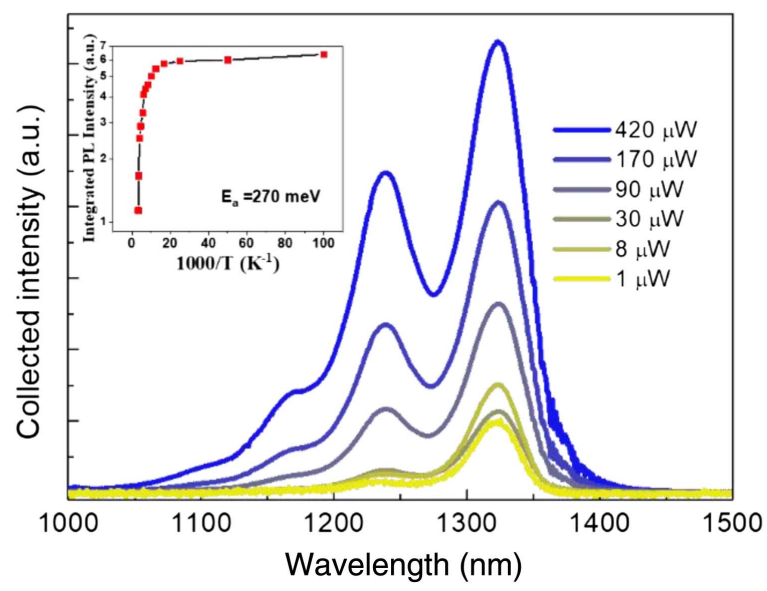

Fig. 1. (a) Schematic epitaxial structure of the active region with total thickness of $\sim 362 \mathrm{~nm}$. (b) A high-resolution TEM image of a single InAs QD. (c) An AFM image of uncapped InAs/GaAs QDs. (d) Collected PL spectra of the asgrown structure under various input optical powers at room temperature. Inset: temperature dependent integrated PL intensity.
$300 \mathrm{~nm}$ GaAs spacer layer is covered at the top with high temperature. Figure 1(a) shows the specific details of the epitaxial structure of the active layer, and four-stack as-grown InAs/InGaAs dot-in-well (DWELL) active layers used as the gain materials were grown on a $1 \mu \mathrm{m} \mathrm{Al}_{0.6} \mathrm{Ga}_{0.4}$ As sacrificial layer ${ }^{[11,12,17]}$, which were sandwiched by two symmetrical $40 \mathrm{~nm} \mathrm{Al} \mathrm{D}_{0.4} \mathrm{Ga}_{0.6}$ As cladding layers. Each stack of DWELL layers consists of 2.7 monolayer InAs QDs grown at $510^{\circ} \mathrm{C}$ on a $2 \mathrm{~nm}$ $\mathrm{In}_{0.15} \mathrm{Ga}_{0.85}$ As layer and capped by a $6 \mathrm{~nm} \operatorname{In}_{0.15} \mathrm{Ga}_{0.85}$ As layer, followed by a $5 \mathrm{~nm}$ low-temperature grown GaAs layer and $45 \mathrm{~nm}$ high-temperature grown GaAs spacer layer at $600^{\circ} \mathrm{C}$. Figure 1(b) illustrates a high-resolution transmission electron microscope (TEM) image of a single QD, with a typical size of $25 \mathrm{~nm}$ in diameter. Figure 1(c) presents uncapped InAs/ GaAs QDs grown on the $\mathrm{Si}(001)$ substrate, which is captured by an atomic force microscope (AFM) with a density of about $4 \times 10^{10} \mathrm{~cm}^{-2}$. The optical property of as-grown QDs is investigated by a micro-photoluminescent ( $\mu$-PL) measurement under room temperature, as presented in Fig. 1(d), showing various PL spectra under different continuous-wave optically pumping powers from $1 \mu \mathrm{W}$ to $420 \mu \mathrm{W}$. A ground state emission of $1322 \mathrm{~nm}$ is observed, accompanying a first excited state of $1240 \mathrm{~nm}$. PL emission from the further excited states of QDs is pumped out while increasing the powers. Furthermore, an integrated PL intensity driven from temperature dependent PL measurement is presented in the inset of Fig. 1(d), showing a thermal activation energy of approximately $270 \mathrm{meV}$, which is fitted by the Arrhenius equation.

Figure 2(a) shows the schematic figure of the photonic crystal bandedge lasers monolithically grown on an on-axis $\mathrm{Si}(001)$ substrate, and the lattice constant $a$ and the air holes diameter $2 r$ are pointed in the inset. The suspended structure is formed by wet etching the sacrificial layer underneath the active layer due to the different etching selection ratios. The purpose of the suspended structure is to better confine the light in the vertical direction, where the active layer is sandwiched by air.

The fabrication processes of this bandedge laser are presented with these steps. First, plasma-enhanced chemical vapor deposition (PECVD) was used for depositing $\sim 120 \mathrm{~nm} \mathrm{SiO} 2$ as a hard mask. Then, $160 \mathrm{~nm}$ Zep520 e-beam resist was spin-coated on the surface, and the pattern of the photonic crystal bandedge cavities array was defined by using electron-beam lithography
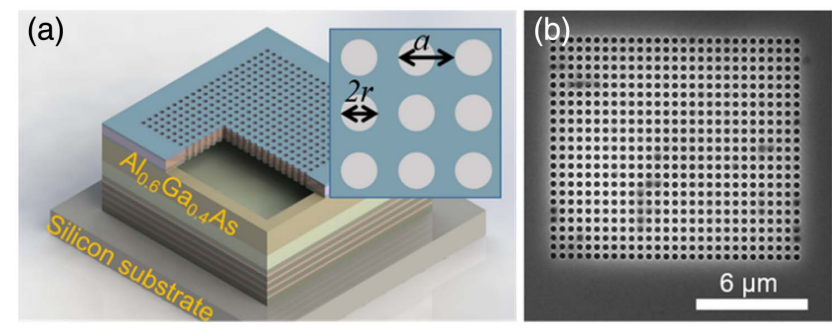

Fig. 2. (a) Schematic diagram of the fabricated InAs/GaAs QD bandedge laser directly grown on an on-axis Si (001) substrate. The lattice constant and radius of air holes are $a$ and $r$, respectively. (b) An SEM image of fabricated photonic crystal bandedge cavity. 


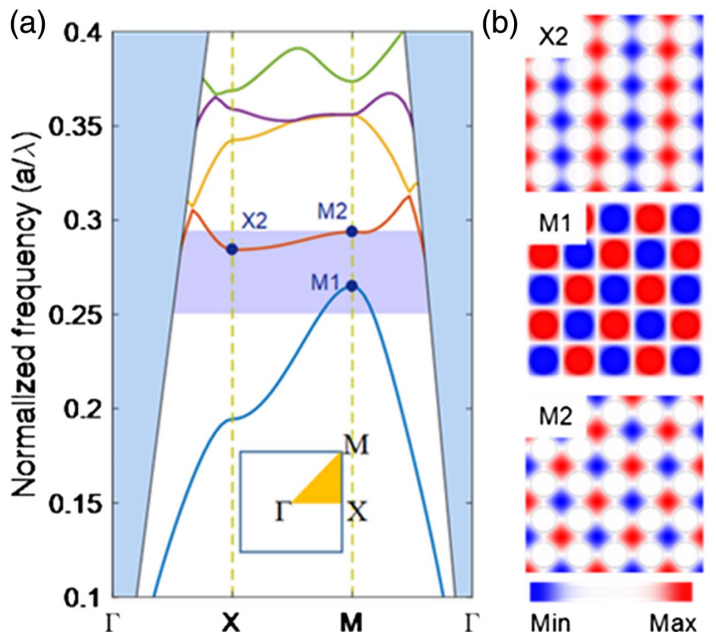

Fig. 3. (a) Dispersion relation diagram of TE-like states for the photonic crystal bandedge cavity with the parameters of $r / a=0.35$ and the slab thickness of 1.05a. The inset shows the irreducible Brillouin zone. The shaded blue region indicates the light cone, and the purple area represents the gain region of asgrown QDs. High symmetry points with low group velocity within the gain region are marked as X2, M1, and $\mathrm{M} 2$. (b) The corresponding calculated hertz $(\mathrm{Hz})$ field profiles.

(EBL) and followed by development. The hard mask is etched by inductively coupled plasma (ICP) reactive ion etching to transfer the pattern. After the removal of the remaining resist, ICP is used for further transformation of the square lattice photonic crystal array to the active region. The wet-etching process was implemented to remove the hard mask and to undercut the sacrificial layer for the formation of a free-standing photonic crystal structure. The fabricated photonic crystal bandedge cavity is shown in Fig. 2(b), which is captured by a scanning electron microscope (SEM).

\section{Discussion}

The dispersion relation diagram and the corresponding eigenfield profiles were calculated by MIT photonic bands (MPBs) based on the plane-wave expansion method ${ }^{[18]}$. The dispersion relation diagram of TE-like states is illustrated in Fig. 3(a), with the bandedge cavity parameters of lattice constant $a=340 \mathrm{~nm}$, $r / a=0.35$, and the slab thickness $\sim 1.05 a$. The light cone is indicated by the blue region. The approximate optical gain region (from $1194 \mathrm{~nm}$ to $1340 \mathrm{~nm}$ ) of the as-grown QDs was represented by the purple region, covering both the ground state and the first excited state obtained by curve fitting of the collected PL spectra. Three bandedge modes at high symmetry points M1, X2, and M2 are possible for the lasing emission because of their locations in the gain region. The corresponding magnetic field profiles of $\mathrm{X} 2, \mathrm{M} 1$, and $\mathrm{M} 2$ are depicted in Fig. 3(b). For further research, to realize single mode emission, some methods can be implemented such as by slightly modifying the parameters of the photonic crystal bandedge cavity to enable only one bandedge point lying within the as-grown
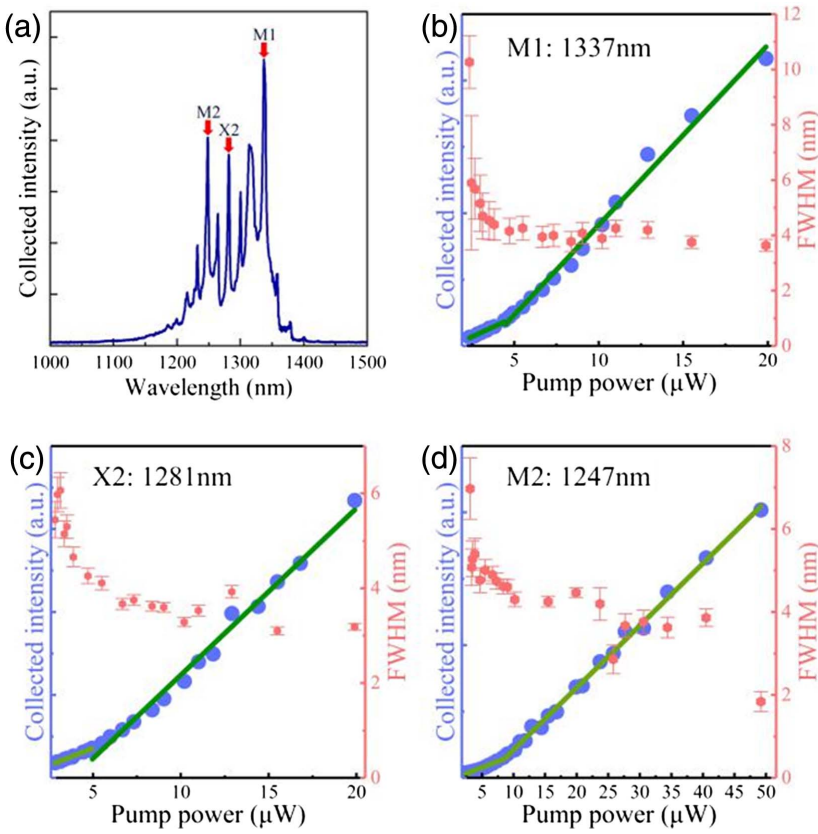

Fig. 4. (a) Emission spectrum of the fabricated photonic crystal bandedge laser, the pump power is $49.2 \mu \mathrm{W}$. Lasing modes of M1, X2, and M2 can be determined by the spectral positions. (b)-(d) The L-L curve and FWHM as a function of input power for three lasing modes $M 1, X_{2}$, and $M 2$, respectively.

InAs QDs gain region for single mode lasing. Introducing the taper region (with linear decrease of air holes) at the $\Gamma$ point to reduce radiation loss at $\mathrm{X} 2$ can also be a convincing way.

A $\mu$-PL system setup was used to characterize the fabricated photonic crystal bandedge lasers. We optically pumped the bandedge cavity at room temperature with a standard $632.8 \mathrm{~nm} \mathrm{He}-$ $\mathrm{Ne}$ gas laser as the pumping source, whose standard Gaussian spot size is around $3 \mu \mathrm{m}$. The PL spectrum of this cavity with $a=340 \mathrm{~nm}$ and $r / a=0.35$ is shown in Fig. 4(a). M2, X2, and M1 points with low group velocity are shown in Fig. 4(a), and the spectral positions of lasing modes are comparable with the calculated normalized frequencies. The light-out light-in (L-L) curves of the three modes are shown in Figs. 4(b)-4(d), with a linewidth narrowing observed. But, it is worth mentioning that due to some imperfection fabrication results, the full width at half maximum (FWHM) $\sim 3 \mathrm{~nm}$ lasing modes are relatively large. The optimization of etching can make the surface of the photonic crystal bandedge cavity smoother, the sidewall of the air holes more vertical, and the diameter of the air holes close to the simulation result, so as to solve the problem of large FWHM. The bandedge modes M1 and X2 emissions peaking at $1337 \mathrm{~nm}$ and $1281 \mathrm{~nm}$ are within the ground state with a lasing threshold around $4 \mu \mathrm{W}$ and $5 \mu \mathrm{W}$, respectively, while the bandedge mode M2 with a peak wavelength of $1247 \mathrm{~nm}$ was in the first excited state, of which the threshold is $\sim 7.5 \mu \mathrm{W}$.

\section{Conclusion}

In conclusion, we have demonstrated InAs/GaAs QD square lattice photonic crystal bandedge lasers monolithically grown on 
on-axis $\mathrm{Si}(001)$ substrates. The lasers operated under continuouswave optical pumping at room temperature. An ultra-low lasing threshold was observed in a multi-mode lasing operation. With further structure design or optimized plasma-etching profiles, a single mode lasing emission is feasible. The demonstrated photonic crystal bandedge lasers monolithically grown on the Si substrate are a promising candidate as a large-scale coherent light source for Si photonics.

\section{Acknowledgement}

This research was supported by the National Natural Science Foundation of China (No. 62174144), Shenzhen Fundamental Research Fund (Nos. JCYJ20210324115605016 and JCYJ20210324120204011), Optical Communication Core Chip Research Platform, Shenzhen Key Laboratory Project (No. ZDSYS201603311644527), Longgang Key Laboratory Project (Nos. ZSYS2017003 and LGKCZSYS2018000015), Longgang Matching Support Fund (Nos. CXPTPT-2017-YJ-002 and 201617486), President's Fund (PF01000154), UK Engineering and Physical Sciences Research Council (EP/ P006973/1 and National Epitaxy Facility), European project H2020-ICT-PICTURE (780930), Royal Academy of Engineering (RF201617/16/28), and French government managed by ANR under the Investissements davenir ANR-10-IRT-05 and ANR-15-IDEX-02 and French RENATECH network.

The devices were manufactured in Core Research Facilities (CRF) at SUSTech, and the authors thank the engineers for their technical support.

${ }^{\dagger}$ These authors contributed equally to this work.

\section{References}

1. D. Thomson, A. Zilkie, J. E. Bowers, T. Komljenovic, G. T. Reed, L. Vivien, D. Marris-Morini, E. Cassan, L. Virot, J.-M. Fédéli, J.-M. Hartmann, J. H. Schmid, D.-X. Xu, F. Boeuf, P. O'Brien, G. Z. Mashanovich, and M. Nedeljkovic, "Roadmap on silicon photonics," J. Opt. 18, 073003 (2016).

2. M. Asghari and A. V. Krishnamoorthy, "Energy-efficient communication," Nat. Photonics 5, 268 (2011).

3. S. Chen, W. Li, J. Wu, Q. Jiang, M. Tang, S. Shutts, S. N. Elliott, A. Sobiesierski, A. J. Seeds, I. Ross, P. M. Smowton, and H. Liu, "Electrically pumped continuous-wave III-V quantum dot lasers on silicon," Nat. Photonics 10, 307 (2016).

4. R. Alcotte, M. Martin, J. Moeyaert, R. Cipro, S. David, F. Bassani, F. Ducroquet, Y. Bogumilowicz, E. Sanchez, Z. Ye, X. Y. Bao, J. B. Pin, and T. Baron, "Epitaxial growth of antiphase boundary free GaAs layer on $300 \mathrm{~mm} \mathrm{Si} \mathrm{(001)} \mathrm{substrate} \mathrm{by} \mathrm{metalorganic} \mathrm{chemical} \mathrm{vapour} \mathrm{deposition}$ with high mobility," APL Mater. 4, 046101 (2016).

5. M. Tang, S. Chen, J. Wu, Q. Jiang, K. Kennedy, P. Jurczak, M. Liao, R. Beanland, A. Seeds, and H. Liu, "Optimizations of defect filter layers for 1.3- $\mu \mathrm{m} \mathrm{InAs/GaAs} \mathrm{quantum-dot} \mathrm{lasers} \mathrm{monolithically} \mathrm{grown} \mathrm{on} \mathrm{Si} \mathrm{sub-}$ strates," IEEE J. Sel. Top. Quantum Electron. 22, 50 (2016).

6. H. Liu, T. Wang, Q. Jiang, R. Hogg, F. Tutu, F. Pozzi, and A. Seeds, "Longwavelength InAs/GaAs quantum-dot laser diode monolithically grown on Ge substrate," Nat. Photonics 5, 416 (2011).

7. A. Lee, Q. Jiang, M. Tang, A. Seeds, and H. Liu, "Continuous-wave InAs/ GaAs quantum-dot laser diodes monolithically grown on $\mathrm{Si}$ substrate with low threshold current densities," Opt. Express 20, 22181 (2012).

8. D. Jung, J. Norman, M. Kennedy, C. Shang, B. Shin, Y. Wan, A. C. Gossard, and J. E. Bowers, "High efficiency low threshold current $1.3 \mu \mathrm{m}$ InAs quantum dot lasers on on-axis (001) GaP/Si," Appl. Phys. Lett. 111, 122107 (2017).

9. Y. Wan, Q. Li, A. Y. Liu, A. C. Gossard, J. E. Bowers, E. L. Hu, and K. M. Lau, "Optically pumped $1.3 \mu \mathrm{m}$ room-temperature InAs quantum-dot micro-disk lasers directly grown on (001) silicon," Opt. Lett. 41, 1664 (2016).

10. T. Zhou, M. Tang, G. Xiang, B. Xiang, S. Hark, M. Martin, M.-L. Touraton, T. Baron, Y. Lu, S. Chen, H. Liu, and Z. Zhang, "Ultra-low threshold InAs/ GaAs quantum dot microdisk lasers on planar on-axis $\mathrm{Si}(001)$ substrates," Optica 11, 430 (2020).

11. T. Zhou, M. Tang, G. Xiang, X. Fang, X. Liu, B. Xiang, S. Hark, M. Martin, T. Baron, S. Pan, J.-S. Park, Z. Liu, S. Chen, Z. Zhang, and H. Liu, "Continuous-wave quantum dot photonic crystal lasers grown on on-axis Si (001)," Nat. Commun. 11, 977 (2020).

12. T. Zhou, M. Tang, H. Li, Z. Zhang, Y. Cui, J.-S. Park, M. Martin, T. Baron, S. Chen, H. Liu, and Z. Zhang, "Single-mode photonic crystal nanobeam lasers monolithically grown on Si for dense integration," IEEE J. Sel. Top. Quantum Electron. 28, 1501906 (2021).

13. H.-Y. Ryu, S.-H. Kwon, Y.-J. Lee, Y.-H. Lee, and J.-S. Kim, "Very-low-threshold photonic band-edge lasers from free-standing triangular photonic crystal slabs," Appl. Phys. Lett. 80, 3476 (2002).

14. S.-H. Kwon, H.-Y. Ryu, G.-H. Kim, Y.-H. Lee, and S.-B. Kim, "Photonic bandedge lasers in two-dimensional square-lattice photonic crystal slabs," Appl. Phys. Lett. 83, 3870 (2003).

15. H.-Y. Lu, S.-C. Tian, C.-Z. Tong, L.-J. Wang, J.-M. Rong, C.-Y. Liu, H. Wang, S.-L. Shu, and L.-J. Wang, "Extracting more light for vertical emission: high power continuous wave operation of $1.3-\mu \mathrm{m}$ quantum-dot photoniccrystal surface-emitting laser based on a flat band," Light Sci. Appl. 8, 108 (2019).

16. S. Noda, K. Kitamura, T. Okino, D. Yasuda, and Y. Tanaka, "Photonic-crystal surface-emitting lasers: review and introduction of modulated-photonic crystals," IEEE J. Sel. Top. Quantum Electron. 23, 4900107 (2017).

17. H. Y. Liu, I. R. Sellers, T. J. Badcock, D. J. Mowbray, M. S. Skolnick, K. M. Groom, M. Gutierrez, M. Hopkinson, J. S. Ng, J. P. R. David, and R. Beanland, "Improved performance of $1.3 \mu \mathrm{m}$ multilayer InAs quantum-dot lasers using a high-growth-temperature GaAs spacer layer," Appl. Phys. Lett. 85, 704 (2004).

18. S. G. Johnson and J. D. Joannopoulos, "Block-iterative frequency-domain methods for Maxwell's equations in a planewave basis," Opt. Express 8, $173(2001)$ 